\title{
Joint Synchronization in Eureka 147 DAB System Based on Abrupt Phase Change Detection
}

\author{
Yung-Liang Huang, Member, IEEE, Chorng-Ren Sheu, Student Member, IEEE, and Chia-Chi Huang
}

\begin{abstract}
We present a joint symbol, frame, and carrier synchronization method for the Eureka 147 DAB signal in this paper. Symbol timing is determined first by detecting an abrupt change in the phase angle of the complex product between the last quarter of a useful symbol and its cyclic extension in the guard interval. The detection of this abrupt change is based on the maximal likelihood (ML) principle. Frequency offset of fractional carrier spacing is estimated from the phase angle of the autocorrelation after symbol timing is estimated. Coarse frame synchronization and null symbol detection can also be achieved through this correlation information. Frequency offset of integral carrier spacing is determined from the convolution outputs between a received phase reference symbol and several locally generated but frequency-shifted phase reference symbols. We found the length of a guard interval is the most important parameter for the synchronization algorithm to work. Simulation results show that the performance of this synchronization method approaches to the ideal synchronization case in both an additive white Gaussian noise (AWGN) channel and a two-path Rayleigh fading channel.
\end{abstract}

Index Terms - Abrupt phase change detection, autocorrelation, digital audio broadcasting (DAB), frame synchronization, frequency offset estimation, guard interval, joint synchronization, orthogonal frequency division multiplexing (OFDM), symbol timing estimation.

\section{INTRODUCTION}

$\mathbf{S}$ YNCHRONIZATIONS at different levels are important in a digital communication system. Demodulation of a received signal usually requires the knowledge of carrier phase, carrier frequency offset (due to oscillator instability or Doppler shift), symbol timing, and frame timing.

Orthogonal frequency division multiplexing (OFDM) transmission was proposed about three decades ago [1]-[3]. Due to the advance in integrated circuits, the fast Fourier transform (FFT) chip replaces the array of sinusoidal modulators and coherent demodulators required in the original multicarrier OFDM system and makes its implementation more feasible.

In order to alleviate the effect of multipath fading, OFDM was adopted by several digital broadcasting systems, such as the European digital audio broadcasting (DAB) system [4], [5] and the digital video broadcasting (DVB) system [6], [7]. Both

Manuscript received October 15, 1998; revised March 3, 1999. This paper was presented in part at the IEEE International Conference on Universal Personal Communications (ICUPC'97), San Diego, CA, Oct. 1997.

Y.-L. Huang is with the Solomon Wireless Tech. Corp., Taipei, Taiwan 300 R.O.C. (e-mail: ylh@solomon.com.tw).

C.-R. Sheu is with National Chiao Tung University, Hsinchu, Taiwan 300 R.O.C. (e-mail: u8513814@cc.nctu.edu.tw).

C.-C. Huang is with the Communication Engineering Department, National Chiao Tung University, Hsinchu, Taiwan 300 R.O.C. (e-mail: huangcc@cc.nctu.edu.tw).

Publisher Item Identifier S 0733-8716(99)07859-2.
DAB and DVB systems are broadband wireless broadcasting systems, and the DAB system can also provide various data services. OFDM is also an attractive candidate for indoor broadband wireless data transmission. HIPERLAN/2 [8], for example, is contemplating the use of OFDM.

In these systems, both carrier synchronization and symbol timing synchronization are difficult receiver design problems. Many synchronization methods have been proposed for OFDM systems. However, they usually either require a special pilot tone structure or solve only part of the synchronization problems [9]-[14]. A complete synchronization system using a DSP approach has been proposed by some authors [15], [16]. Here, we propose a synchronization method that jointly completes frame, carrier, and symbol synchronization and is applicable to any OFDM transmission with a cyclicly extended guard interval. The synchronization method is suitable for an application specific integrated circuit (ASIC) implementation due to its all digital nature [17].

In Section II, we first discuss the impacts on OFDM system performance from synchronization defects, including frequency offset and symbol timing offset. In Section III, we present our synchronization method in terms of block diagrams and describe some detailed signal processing procedures. The performance of our synchronization method is evaluated by computer simulation, and the results are presented in Section IV. Finally, we give a brief conclusion in Section V.

\section{SYSTEM IMPACTS FROM SYNCHRONIZATION DEFECTS}

\section{A. Impacts from Frequency Offset}

A Eureka 147 OFDM symbol consists of $N$ sinusoidal carriers with frequency spacing $1 / T_{u}$ ( $T_{u}$ : useful symbol period), and each carrier is differential quatenary phase shift key (DQPSK) modulated on a symbol-by-symbol basis. Although the spectra of the modulated carriers overlap, they are orthogonal, i.e., without intercarrier interference (ICI). Nevertheless, a carrier frequency offset between a transmitter and receiver easily destroys the orthogonality, introduces ICI, and causes errors in the detection of an OFDM signal.

During a useful symbol period $T_{u}$, the complex envelope of a transmitted OFDM signal can be expressed as

$$
\begin{aligned}
x_{n} & =\frac{1}{N} \sum_{k=-K / 2}^{K / 2} z_{k} \exp \left\{j 2 \pi k f_{\mathrm{scs}} n T_{s}\right\} \\
& =\frac{1}{N} \sum_{k=-K / 2}^{K / 2} z_{k} \exp \left\{j \frac{2 \pi k n}{N}\right\}
\end{aligned}
$$


since

$$
N f_{\mathrm{scs}}=\frac{1}{T_{s}}
$$

where $T_{s}$ is the sampling period, $N$ is the number of FFT samples per symbol $\left(T_{u}=N T_{s}\right), K$ is the number of active channels, $z_{k}$ is the complex data symbol modulating the $k$ th active channel, and $f_{\text {scs }}$ is the subcarrier spacing. We note that $N$ is larger than $K$, and it is typically chosen as a power of two.

Following the same notation as in [18], we define

$$
\tilde{z}_{k}=\sum_{r=-\infty}^{\infty} z_{k+r N}
$$

and

$$
\Re_{N}[k] \equiv \begin{cases}1, & 0 \leq k \leq N-1 \\ 0, & \text { otherwise. }\end{cases}
$$

With this definition we have from (1)

$$
x_{n}=\frac{1}{N} \sum_{k=0}^{N-1} \tilde{z}_{k} \Re_{N}[k] \exp \left\{j \frac{2 \pi k n}{N}\right\} .
$$

With a frequency offset $\Delta f$ and ignoring noise, the received complex envelope will be

$$
\begin{aligned}
y_{n} & =\frac{1}{N} \sum_{k=0}^{N-1} \tilde{z}_{k} \Re_{N}[k] H_{k} \exp \left\{j 2 \pi(k+\delta) f_{\mathrm{scs}} n T_{s}\right\} \\
& =\frac{1}{N} \sum_{k=0}^{N-1} \tilde{z}_{k} \Re_{N}[k] H_{k} \exp \left\{j \frac{2 \pi(k+\delta) n}{N}\right\}
\end{aligned}
$$

where $H_{k}$ is the corresponding cyclicly-shifted transfer function of the channel at the frequency of the $k$ th subcarrier, and $\delta=\Delta f / f_{\mathrm{scs}}$ is the normalized frequency offset.

Assuming perfect clock recovery and no noise, the $N$ point FFT of $y_{n}$ becomes

$$
\begin{aligned}
Y_{l}= & \sum_{n=0}^{N-1} y_{n} \exp \left\{-j \frac{2 \pi l n}{N}\right\} \\
= & \sum_{n=0}^{N-1}\left[\frac{1}{N} \sum_{k=0}^{N-1} \tilde{z}_{k} \Re_{N}[k] H_{k} \exp \left\{j \frac{2 \pi(k+\delta) n}{N}\right\}\right] \\
& \cdot \exp \left\{-j \frac{2 \pi l n}{N}\right\} \\
= & \frac{1}{N} \sum_{k=0}^{N-1} \tilde{z}_{k} \Re_{N}[k] H_{k} \sum_{n=0}^{N-1} \exp \left\{j \frac{2 \pi(k+\delta) n-2 \pi l n}{N}\right\} \\
= & \frac{1}{N} \sum_{k=0}^{N-1} \tilde{z}_{k} \Re_{N}[k] H_{k} \sum_{n=0}^{N-1} \exp \left\{j \frac{2 \pi[(k+\delta)-l] n}{N}\right\} .
\end{aligned}
$$

When $\delta$ is an integer, we have

$$
Y_{l}=\tilde{z}_{l-\delta} \Re_{N}[l-\delta] H_{l-\delta} .
$$

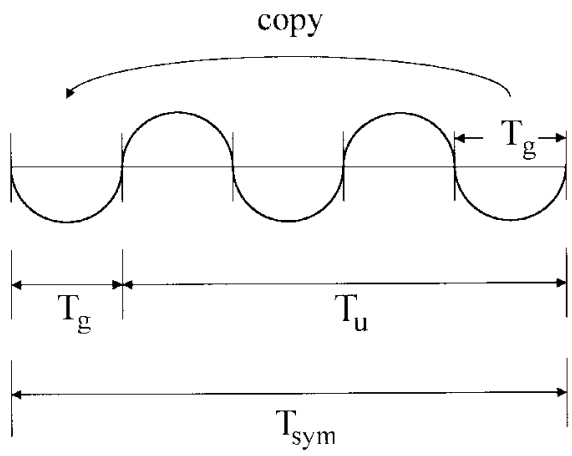

Fig. 1. Cyclic extension of an OFDM symbol. $T_{\text {sym }}$ : symbol period. $T_{u}$ : useful symbol period. $T_{g}$ : guard period.

That is, the entire spectrum of an OFDM signal is cyclicly shifted, and there is no ICI.

Now, we consider the case in which $\delta$ is a real number with absolute value less than one. Since

$$
\begin{aligned}
\sum_{n=0}^{N-1} \exp \left\{j \frac{2 \pi[(k+\delta)-l] n}{N}\right\} & \frac{\sin \{\pi[(k+\delta)-l]\}}{\sin \left\{\frac{\pi[(k+\delta)-l]}{N}\right\}} \\
& \cdot \exp \left\{j \frac{\pi[(k+\delta)-l](N-1)}{N}\right\} \\
Y_{l}= & \frac{1}{N} \tilde{z}_{l} \Re_{N}[l] H_{l} \frac{\sin [\pi \delta]}{\sin \left[\frac{\pi \delta}{N}\right]} \exp \left\{j \frac{\pi \delta(N-1)}{N}\right\} \\
& +\frac{1}{N} \sum_{\substack{k=0 \\
k \neq l}}^{N-1} \tilde{z}_{z} \Re_{N}[k] H_{k}(-1)^{k-l} \frac{\sin [\pi \delta]}{\sin \left[\frac{\pi(k-l+\delta)}{N}\right]} \\
& \cdot \exp \left\{j \frac{\pi(k-l+\delta)(N-1)}{N}\right\} \\
= & \hat{x}_{l}+I_{l}
\end{aligned}
$$

where $I_{l}$ is the ICI. We observe that the magnitude of the desired signal $\hat{x}_{l}$ is affected by both $H_{l}$ and $\sin [\pi \delta] / \sin [\pi \delta / N]$. Besides, the term $\exp \{j(\pi \delta(N-1) / N)\}$ introduces a constant phase shift that can be neglected in differential demodulation. The smaller $\delta$ is, the smaller ICI is. In order to achieve a signal-to-ICI power ratio of at least $100(20 \mathrm{~dB}), \delta$ must be less than $5 \%[16]$.

\section{B. Impacts from Symbol Timing Offset}

To solve symbol timing offset problems [19], a guard interval is introduced in front of each Eureka 147 OFDM symbol. The guard interval contains a periodic extension of the useful symbol itself, as shown in Fig. 1. The effect of symbol timing offset on system performance can be totally eliminated if the offset is smaller than the length of the guard interval in a channel without multipath. As long as the time reference begins in the guard interval, we get a cyclicly shifted symbol. The cyclic shift in the time domain results in a linear phase shift in the frequency domain [18]. 


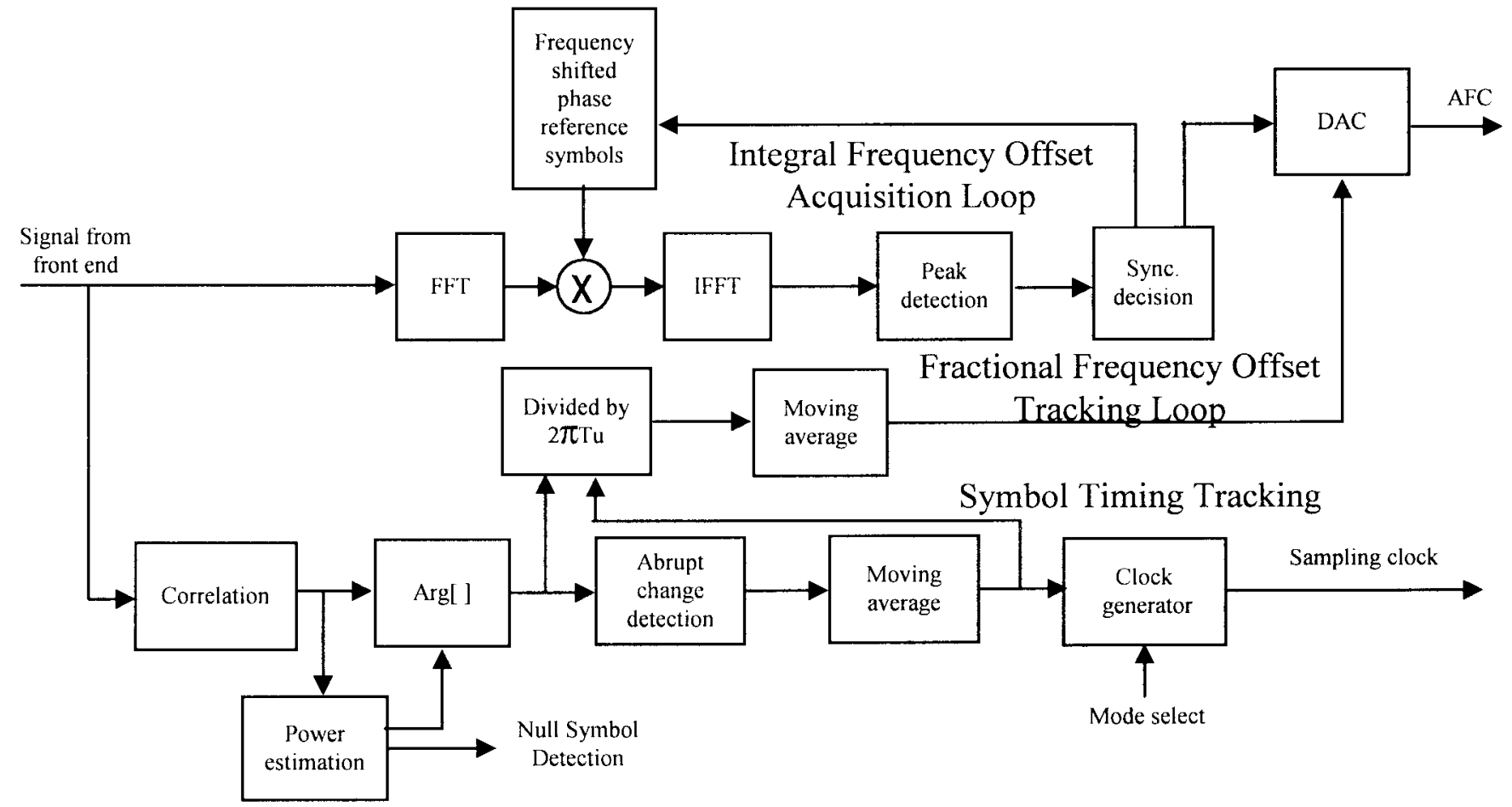

Fig. 2. The block diagram of our synchronization method. Tu: useful symbol period.

The effect of this linear phase shift can be neglected when differential detection techniques are used. Since symbol-bysymbol DQPSK modulation is adopted in the Eureka 147 DAB system, there is no problem in demodulating a received OFDM signal as long as the time reference begins in the guard interval.

Another purpose of the guard interval insertion is to minimize the effect of multipath. This effect causes a form of linear distortion. In general, the length of the guard interval should be longer than the multipath delay spread. There will be no degradation in system performance, provided that the time reference begins in the intersymbol interference- (ISI) free portion of the guard interval [19].

\section{A NEW SYNCHRONIZATION METHOD FOR OFDM SYSTEMS}

The block diagram of our synchronization method is shown in Fig. 2. The blocks in the figure can be implemented in ASIC. The "correlation" block and the "abrupt change detection" block are shown in more detail in Fig. 3.

\section{A. Symbol Timing Estimation}

A typical output of the Arg[ ] block in Fig. 2 is shown in Fig. 4(a). The one-sample differentiator in Fig. 3 makes its output in the last $T_{g}$ period (this period is denoted as $\Gamma$ for later use) of a symbol close to zero. In fact, the output has two different distributions with the same (zero) mean, but different variances in different parts of the OFDM signal. One is uniformly distributed between $[-\pi, \pi]$ with zero mean and constant variance $\pi^{2} / 3$. The other is Gaussian distributed with zero mean and variance $\sigma^{2}$, which depends on the noise level. Therefore, we can detect the OFDM symbol timing by detecting the abrupt change in the variance.

The probability density functions of two distributions are

$$
\begin{aligned}
& p_{H_{0}}(y)=\frac{1}{2 \pi} \\
& p_{H_{1}}(y)=\frac{1}{\sigma \sqrt{2 \pi}} \exp \left\{-\frac{y^{2}}{2 \sigma^{2}}\right\}
\end{aligned}
$$

where the indexed hypothesis $H_{0}$ holds for the beginning $T_{u}$ period of an OFDM symbol, and $H_{1}$ holds for the last $T_{g}$ period of the symbol. A log-likelihood ratio $S_{i}$ is defined to be

$$
\begin{aligned}
S_{i} & =\ln \frac{p_{H_{1}}\left(y_{i}\right)}{p_{H_{0}}\left(y_{i}\right)} \\
& =\ln \left[\frac{\sqrt{2 \pi}}{\sigma} \exp \left\{-\frac{y_{i}^{2}}{2 \sigma^{2}}\right\}\right] \\
& =C-\frac{y_{i}^{2}}{2 \sigma^{2}}
\end{aligned}
$$

and a decision function can be defined as

$$
S_{1}^{M}=\frac{1}{2 \sigma^{2}} \sum_{i=1}^{M} y_{i}^{2}
$$

where $S_{1}^{M}$ is negatively related to the log-likelihood ratio for the observations from $y_{1}$ to $y_{M}$.

At a fixed sample size $M$, the optimal decision rule is given by

$$
\begin{aligned}
& H_{0} \text { is chosen, if } S_{1}^{M} \geq h \\
& H_{1} \text { is chosen, if } S_{1}^{M}<h
\end{aligned}
$$




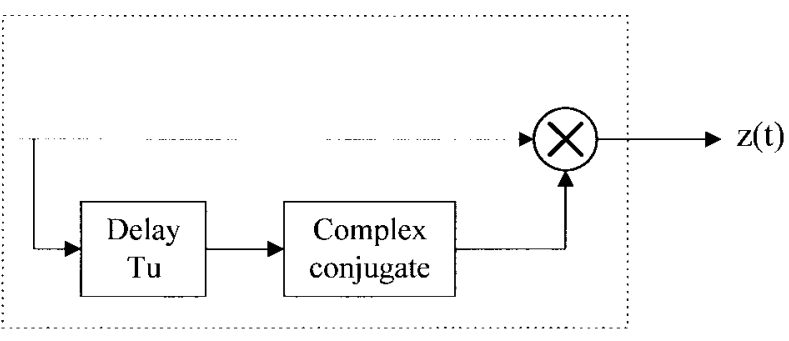

\section{Correlation}

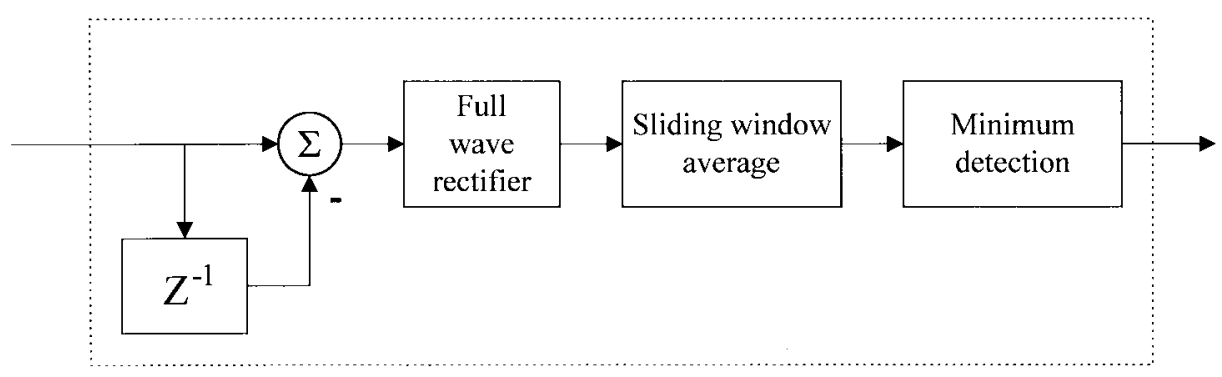

Abrupt change detection

Fig. 3. More details of the "correlation" block and the "abrupt change detection" block in Fig. 2.

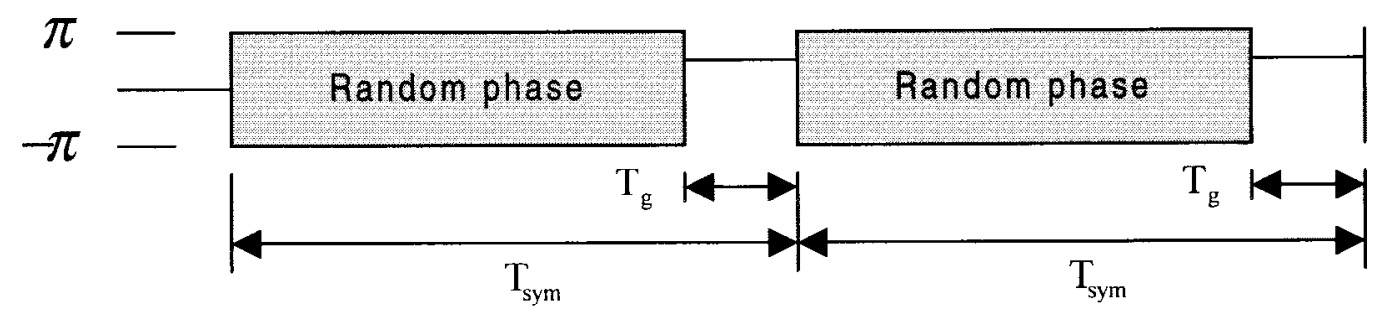

(a)

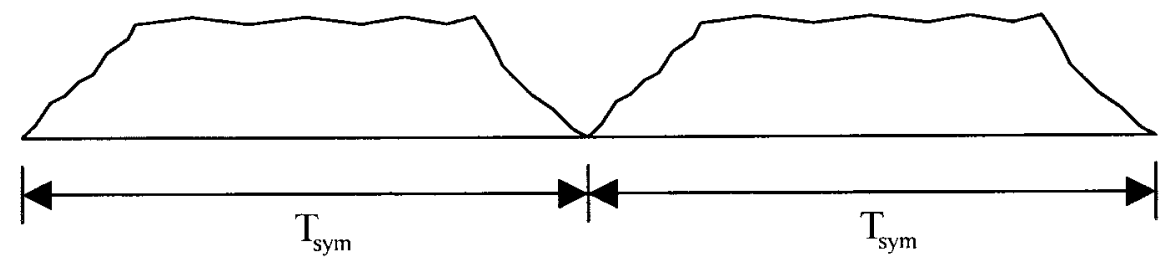

(b)

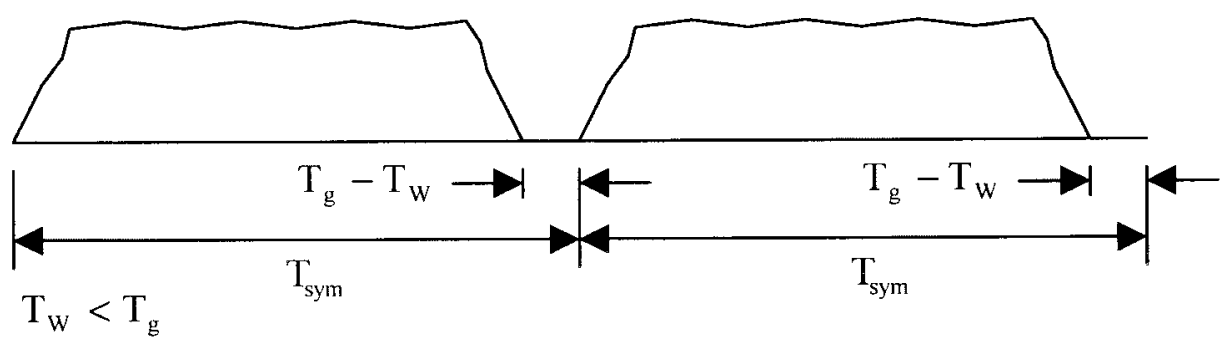

(c)

Fig. 4. (a) Variation in the phase of $z(t)$ and the corresponding symbol timing. (b) Moving average output with window length $T w$ equal to $T g$. (c) Moving average output with window length $T w$ less than $T g$.

where $h$ is a threshold that can be determined by the variances of the two distributions in (10) and the sample size $M$. $\sum_{i=1}^{M} y_{i}^{2}$ can be changed to $\sum_{i=1}^{M}\left|y_{i}\right|$ for easy implementation. This is the reason why a full-wave rectifier is used in Fig. 3.
In Fig. 3, we do a sliding window average, i.e., implement the decision function and generate output signals, as shown in Fig. 4(b) or (c). With signals, as shown in Fig. 4(b) or (c), we can detect the desired symbol timing. It is well-known that the threshold should depend on noise level. For purposes of 
simplicity, we detect the minimum of the moving-averaged outputs and denote its location as the desired symbol timing. If several minima are detected, the arithmetic mean of the corresponding locations of the first minimum and the last one is denoted as the symbol timing. This symbol timing is moving-averaged afterwards. Besides, we use the averaged timing as a time reference to calculate frequency offset within a window around this time reference, as shown in Fig. 2.

\section{B. Frequency Offset Estimation}

We assume a frequency offset $\Delta f=\left(\delta_{\mathrm{in}}+\delta_{\mathrm{fr}}\right) f_{\mathrm{scs}}$ exists, where $\delta_{\text {in }}$ is an integer and $\delta_{\mathrm{fr}}$ is a real number with absolute value less than one. When the received signal $r(t)$ reaches the last $T_{g}$ period of a symbol, we have samples of $z(t)$ at the output of the "Correlation" block in Fig. 3, where

$$
\begin{aligned}
z(t)= & r(t) r^{*}\left(t-T_{u}\right) \\
= & a(t) \exp \left\{j 2 \pi\left(\delta_{\mathrm{fr}}+\delta_{\mathrm{in}}\right) f_{\mathrm{scs}} t\right\} a^{*}\left(t-T_{u}\right) \\
& \cdot \exp \left\{-j 2 \pi\left(\delta_{\mathrm{fr}}+\delta_{\mathrm{in}}\right) f_{\mathrm{scs}}\left(t-T_{u}\right)\right\} \\
= & c(t) \exp \left\{j 2 \pi \delta_{\mathrm{fr}} f_{\mathrm{scs}} T_{u}\right\} \\
= & c(t) \exp \left\{j 2 \pi \delta_{\mathrm{fr}}\right\} .
\end{aligned}
$$

In (14), $a(t)$ is the received symbol when frequency offset is zero, and $c(t)$ is defined as

$$
c(t) \equiv a(t) a^{*}\left(t-T_{u}\right) \approx a(t) a^{*}(t)=|a(t)|^{2}>0 .
$$

The approximation holds because we assume the OFDM symbol rate is much higher than the channel fading rate. We can estimate $\delta_{\text {fr }}$, the normalized fractional frequency offset from

$$
\delta_{\mathrm{fr}}=\frac{\overline{\arg \left[z\left(n T_{s}\right)\right]}}{2 \pi} .
$$

In the last $T_{g}$ period of a symbol, $\arg \left[z\left(n T_{s}\right)\right]$ is almost a constant and is proportional to $\delta_{\mathrm{fr}}$. Elsewhere, $\arg \left[z\left(n T_{s}\right)\right]$ will have random values uniformly distributed between $[-\pi, \pi]$. Fig. 4(a) shows the phenomenon.

The abrupt change of this phase distribution provides the symbol timing information, as described in the previous section, and it can be used to start and stop the calculation of $\delta_{\mathrm{fr}}$ in (16). We use the averaged timing as a reference to calculate frequency offset using the ten samples of $\arg \left[z\left(n T_{s}\right)\right]$ around this reference. In this way, the calculation of frequency offset is inherently free of multipath (which introduces random phase) and automatically adapts to different multipath delay spreads. As we calculate frequency offset only in the periods free of multipath distortion, our algorithm is robust in a mobile radio environment. The receiver fractional frequency offset is afterwards compensated by an automatic frequency control (AFC) circuit in the front end.

\section{Null Symbol Detection}

In the Eureka 147 DAB system, a null symbol precedes a phase reference symbol in an OFDM frame. During the null symbol period, no signal is transmitted. That is, the $c(t)$ in (15) will be equal to zero when noise does not exist. Thus, a power estimation circuit, e.g., an envelope detector as shown in Fig. 2, can be used to detect the occurrence of an OFDM frame. The frame timing can thus be detected if the received signal power falls below a predetermined threshold within an appropriately chosen observation window.

It is well-known that an OFDM signal has a large peakto-average power ratio. When the instantaneous power of an OFDM signal is very low, the phase angle of the correlation output $z(t)$ could be distorted by noise. In order to reduce this noise problem, we suggest to hold the phase detector output, i.e. the $\arg \prod$ block output, to the previous output when the signal power falls below a predetermined threshold.

\section{Integral Frequency Offset Estimation}

An OFDM symbol is treated as a complex vector here. Assuming there are $M$ prestored vectors $\mathbf{x}_{i}, i=1,2, \ldots, M$ and an input vector $\mathbf{y}$, we want to find the $\mathbf{x}_{i}$ which is most similar to $\mathbf{y}$. Without loss of generality, the vectors $\mathbf{x}_{i}$ and the input vector $\mathbf{y}$ are normalized such that

$$
\left\|\mathbf{x}_{i}\right\|=1
$$

for $i=1,2, \ldots, M$, and

$$
\|\mathbf{y}\|=1
$$

where $\|\mathrm{x}\|$ is the norm of the vector $\mathbf{x}$ and is defined as

$$
\|\mathrm{x}\|=\sqrt{\sum_{k=1}^{N}\left|x_{k}\right|^{2}} .
$$

The square of the distance between two vectors becomes

$$
\begin{aligned}
\left\|\mathbf{y}-x_{i}\right\|^{2} & =\|\mathbf{y}\|^{2}-2\left(\mathbf{x}_{i} \cdot \mathbf{y}\right)+\left\|\mathbf{x}_{i}\right\|^{2} \\
& =2-2\left(\mathbf{x}_{i} \cdot \mathbf{y}\right) \\
& =2-2 \cos \theta
\end{aligned}
$$

where $\theta$ is the angle between the two vectors. The common implementation of the maximum inner product rule is known as a matched filter or a correlator.

Since the phase reference symbol (PRS) [4] in Eureka 147 has the property that it is orthogonal to itself with any integral frequency offset (IFO), IFO can be estimated by a matched filter using the received but fractional frequency offset- (FFO) compensated PRS. The FFO-compensated PRS $(\mathbf{y})$ is matched to several prestored local PRS's $\left(\mathrm{x}_{i}\right)$ with different IFO's. The one that most closely matches the FFOcompensated PRS produces peaks in its output (the peak output can be compared with a predetermined threshold to determine whether the system is in synchronization or not), while the others produce noise-like outputs. Thus, IFO can be identified and compensated. Since a practical oscillator has frequency stability in the order of several ppm, only a few candidate PRS's are required.

The matched filtering (or convolution) operation in the time domain can be converted into multiplication in the frequency domain by FFT to reduce computation complexity. As shown in Fig. 2, both FFT and inverse fast Fourier transform (IFFT) calculations can be performed by an ASIC chip. 


\section{E. A Complete Synchronization Procedure}

A complete synchronization procedure for the Eureka 147 DAB system is proposed in this section. When a receiver is turned on, it first estimates symbol timing and FFO $\left(\delta_{\text {fr }}\right)$. Then it compensates for $\delta_{\mathrm{fr}}$. Simultaneously, it detects the occurence of the null symbol (or frame timing). Finally, it uses an FFOcompensated PRS to estimate IFO $\left(\delta_{\text {in }}\right)$ and compensates for $\delta_{\text {in }}$.

The complete synchronization procedure consists of both acquisition and tracking stages. For acquisition (on power up or loss of track), perform the following steps.

A1) Acquire symbol timing.

A2) Calculate $\delta_{\text {fr }}$ and compensate for it.

A3) Detect the null symbol and the frame timing.

A4) Detect the integral frequency offset using the the integral frequency offset acquisition loop (see Fig. 2).

A5) Go to tracking if the system is in synchronization. Otherwise, go back to A1.

For tracking (after successful acquisition), perform the following steps.

T1) Track symbol timing (see Fig. 2).

T2) Track $\delta_{\text {fr }}$ using the fractional frequency offset tracking loop (see Fig. 2).

\section{Simulation Results}

In this section, we demonstrate the performance of our synchronization algorithm on symbol timing offset estimation, frequency offset estimation, and system BER performance. The algorithm is investigated using the Monte-Carlo simulation method in both an additive white Gaussian noise (AWGN) channel and a two-path fading channel. We assumed the mode 3 OFDM signaling is used in the Eureka 147 DAB system [4] for the simulation. In this scenario, the overall symbol duration is $156 \mu \mathrm{s}$, which includes a useful symbol duration of $125 \mu \mathrm{s}$ and a guard interval duration of $31 \mu \mathrm{s}$. There are 192 active subcarriers with an $8 \mathrm{KHz}$ inter-carrier spacing in total.

In our simulation, we get one estimated symbol timing sample and ten estimated frequency offset samples per symbol. Both the estimated symbol timing samples and the estimated frequency offset samples are moving-averaged with 200 samples. We simulated 5000 symbols in the AWGN channel and 12000 symbols in the two-path fading channel to obtain both the means and the standard deviations of these estimations.

\section{A. AWGN Channel}

1) Results on Symbol Timing Estimation: We define symbol timing offset as a right timing offset (RTO) when sampling time lags the guard interval, and we define symbol timing offset as a left timing offset (LTO) when sampling time leads the guard interval. The effect of RTO and LTO should be the same. As long as sampling time is within the guard interval, there should be no error in OFDM demodulation, assuming there is no noise or any other impairments.

From our simulation, the effect of symbol timing offset on bit-error rate (BER) performance of an OFDM system in an AWGN channel is shown in Fig. 5. Here, only raw bit error

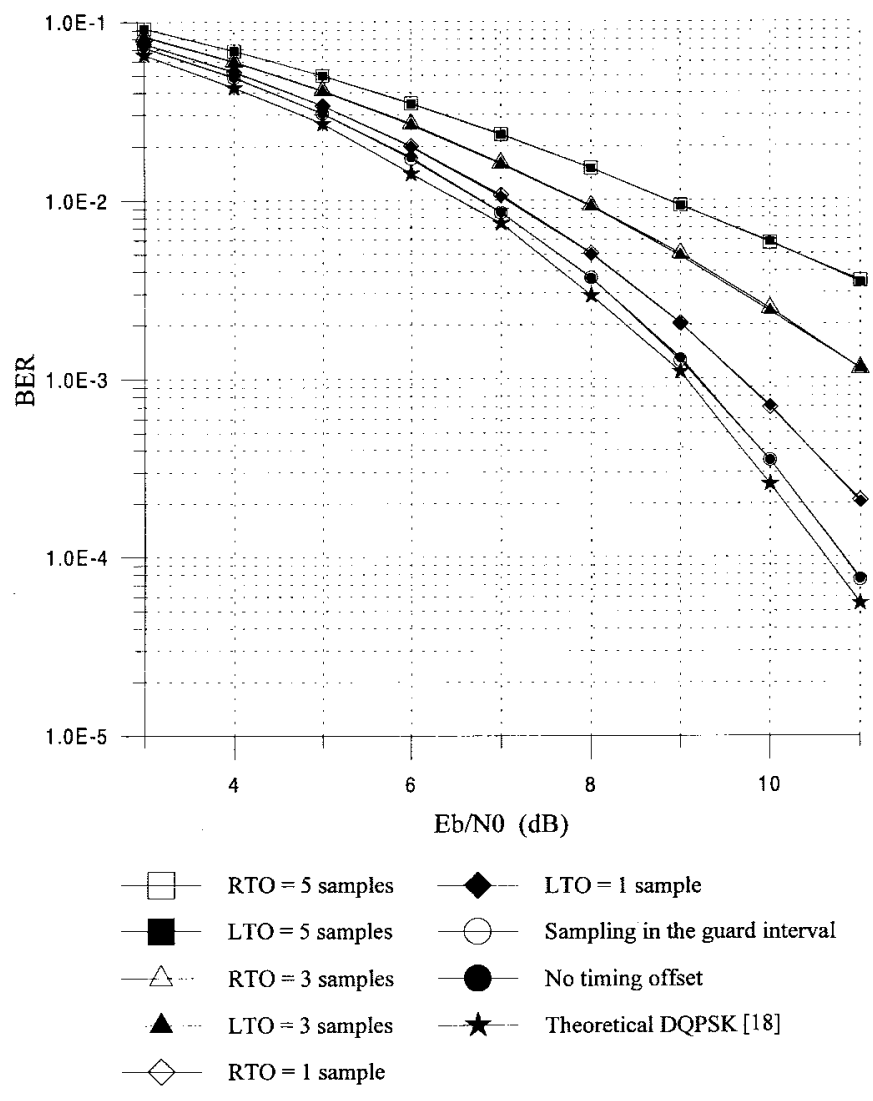

Fig. 5. BER performance versus timing offset in an AWGN channel.

without coding and interleaving is measured. Theoretical BER performance of DQPSK [21, Fig. 5-2-13, p. 277] is shown for calibration purposes. We observe that as long as the sampling time is in the guard interval, there is no BER degradation, as compared with the perfect timing case. On the other hand, if the initial sampling time is out of the guard interval, even offset by only one sample, performance degradation occurs. The BER is almost the same for the same RTO and LTO values.

Simulation results in Table I show the means $(\hat{m}$ and $\tilde{m}$, defined later) and the standard deviation (STD) of timing estimation in an AWGN channel. $\hat{m}$ is the mean of an initial timing estimation. Because the sliding window average operation in Fig. 2 causes a delay of half of the sliding window size (SWS) of the samples, this delay should be substracted from $\hat{m}$ to generate the time reference $\tilde{m}$, i.e., $\tilde{m}=\hat{m}-\frac{1}{2}$ SWS. In our simulation, the region $\Gamma$ is between the ninety sixth and the one hundred and fifty eighth samples. $\Omega$ is the ISI free region within $\Gamma . \Omega=(a, b)$ means that the ISI-free region is between the $a$ th and the $b$ th sample. In an AWGN channel, $\Omega=\Gamma$. We define two tolerances, $\Delta_{l}$ and $\Delta_{r}$, as the deviation (normalized by STD) from $\tilde{m}$ to the two limits of $\Omega$, i.e., $\Delta_{l}=(\tilde{m}-a) / \mathrm{STD}, \Delta_{r}=(b-\tilde{m}) / \mathrm{STD}$. From this point of view, our method performs well because it can tolerate at least ten times the STD even when $E_{b} / N_{0}$ is down to $4 \mathrm{~dB}$.

2) Results on Frequency Offset Estimation: The effect of different frequency offsets on OFDM-BER performance was simulated. The results are shown in Fig. 6 for an 
TABLE I

Symbol Timing Estimation Results in an AWgn Channel. (Note: From the Ninety Sixth to One Hundred and Fifty Eighth Sample is Assumed.) SWS $=63$ SAmples. $\boldsymbol{\Omega}=96158$

\begin{tabular}{|c|c|c|c|c|c|}
\hline $\mathrm{E}_{\mathrm{b}} / \mathrm{N}_{0}(\mathrm{~dB})$ & 4 & 6 & 8 & 10 & 12 \\
\hline (i-th sample) & 158 & 158 & 158 & 158 & 158 \\
\hline$\underset{\text { (i-th sample) }}{\widetilde{m}}$ & 126.5 & 126.5 & 126.5 & 126.5 & 126.5 \\
\hline$\underset{\text { (samples) }}{\text { STD }}$ & 3 & 3 & 3 & 3 & 3 \\
\hline & $10.2,10.5$ & $10.2,10.5$ & $10.2,10.5$ & $10.2,10.5$ & $10.2,10.5$ \\
\hline
\end{tabular}
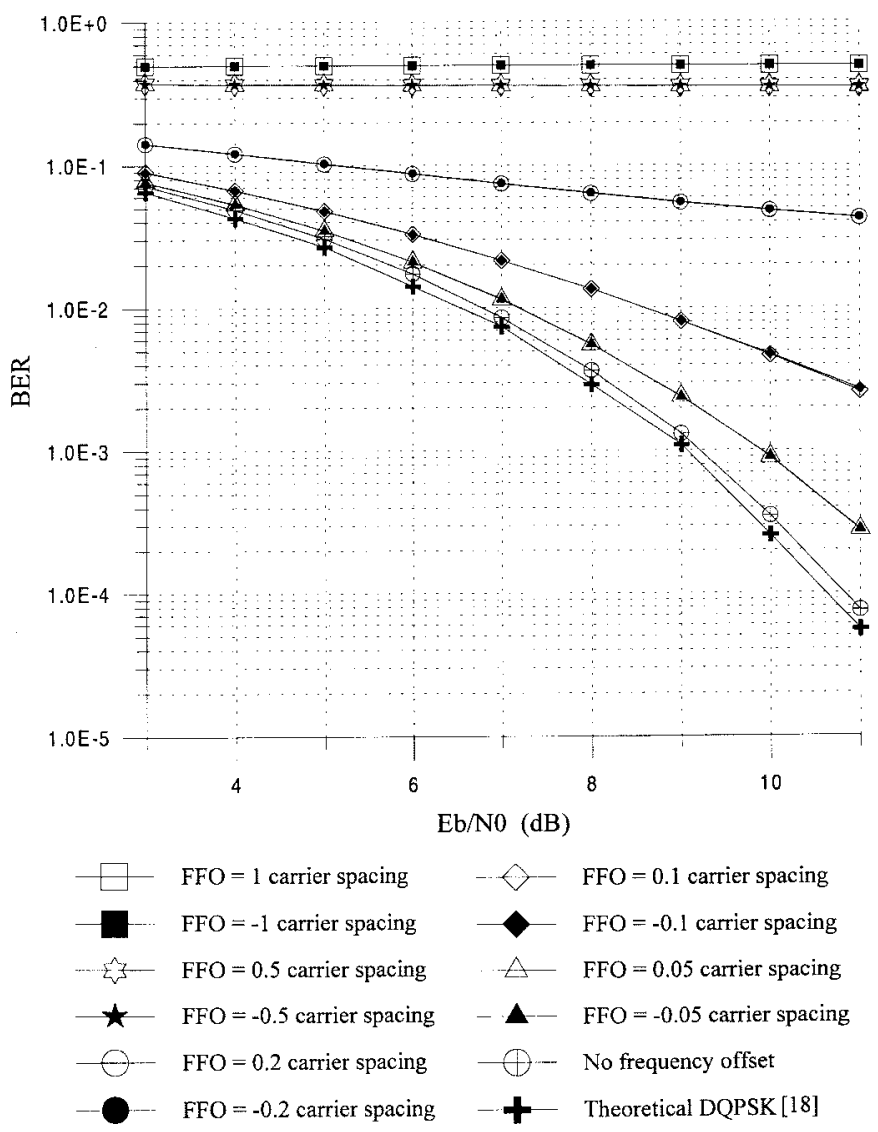

Fig. 6. BER performance with frequency offset as a parameter in an AWGN channel.

AWGN channel. We observe that larger frequency offsets cause more BER degradation due to ICI. When frequency offset approaches 0.5 subcarrier spacing, the average BER approaches 0.5 . On the other hand, the degradation is small if the normalized frequency offset (with respect of subcarrier spacing) is less than 5\%. The sign of FFO does not influence the BER performance.

In the following, we investigate the effect of FFO only and assume IFO can be easily estimated and compensated. Two sets of FFO are used in our computer simulation. The first set is \pm 0.49 , which represents a more critical condition of ICI. The other set is \pm 0.1 , which represents a less critical condition of ICI. The mean and the STD of fractional frequency offset estimation results in an AWGN channel are listed in Table II and plotted in Fig. 7, respectively. It can be observed from Fig. 7 that our algorithm achieves a much better accuracy than the needed 0.05 subcarrier spacing in an AWGN channel, even when $E_{b} / N_{0}$ is down to $4 \mathrm{~dB}$.

Other authors [22] have shown that BER is sensitive to carrier frequency offset in OFDM systems. The effect of our synchronization algorithm on BER (raw BER obtained without convolutional encoding) performance of the mode 3 Eureka 147 system in an AWGN channel is shown in Fig. 8. Symbol timing can always be estimated correctly and is not a problem in these simulations. It can be seen that the BER performance with our synchronization algorithm almost coincides with the perfect synchronization case in an AWGN channel. The effectiveness of our synchronization algorithm can also be seen when comparing Fig. 8 with Fig. 6.

\section{B. Two-Path Fading Channel}

Our synchronization algorithm was simulated using a twopath Rayleigh fading channel model. We assume the two paths have equal strength, and each path is a Rayleigh fading path. The delay between the two paths is a simulation parameter varying from 5-30 $\mu$ s. The two-path Rayleigh fading channel model is one of many different channel models which can be used to simulate the Eureka 147 DAB system. We use this model because it is simple, and it represents a typically severe channel condition.

1) Results on Symbol Timing Estimation: Symbol timing estimation results in a two-path fading channel at a Doppler shift of $240 \mathrm{~Hz}$ are shown in Fig. 9 and Table III. Note that the estimated symbol timing is still valid as long as the time reference $\tilde{m}$ is in the multipath-free region of $\Gamma$. Table III shows the corresponding $\tilde{m}$, STD $, \Delta_{l}, \Delta_{r}$, and $\Omega$ defined in Section IV-A1. From Table III, it can be observed that the timing estimation accuracy is inversely proportional to multipath delay and is not sensitive to SWS. When average signal-to-noise ration (SNR) is $13 \mathrm{~dB}$, we can obtain an accuracy timing estimation even when the multipath delay is $20 \mu \mathrm{s}$.

2) Results on Frequency Offset Estimation: The effect of different frequency offsets on OFDM-BER performance is simulated for a two-path fading channel. Coding and interleaving are applied for completeness. 
TABLE II

The Mean of Frequency Offset Estimation in an AWGN Channel

\begin{tabular}{c|c|c|c|c|c}
\hline$\delta_{f r} \mathrm{E}_{\mathrm{b}} / \mathrm{N0}(\mathrm{dB})$ & 4 & 6 & 8 & 10 & 12 \\
\hline 0.49 & 0.492 & 0.491 & 0.491 & 0.491 & 0.49 \\
\hline 0.1 & 0.0841 & 0.0893 & 0.093 & 0.0954 & 0.097 \\
\hline-0.1 & -0.0845 & -0.0898 & -0.0934 & -0.0956 & -0.0973 \\
\hline-0.49 & -0.492 & -0.491 & -0.491 & -0.49 & -0.49 \\
\hline
\end{tabular}
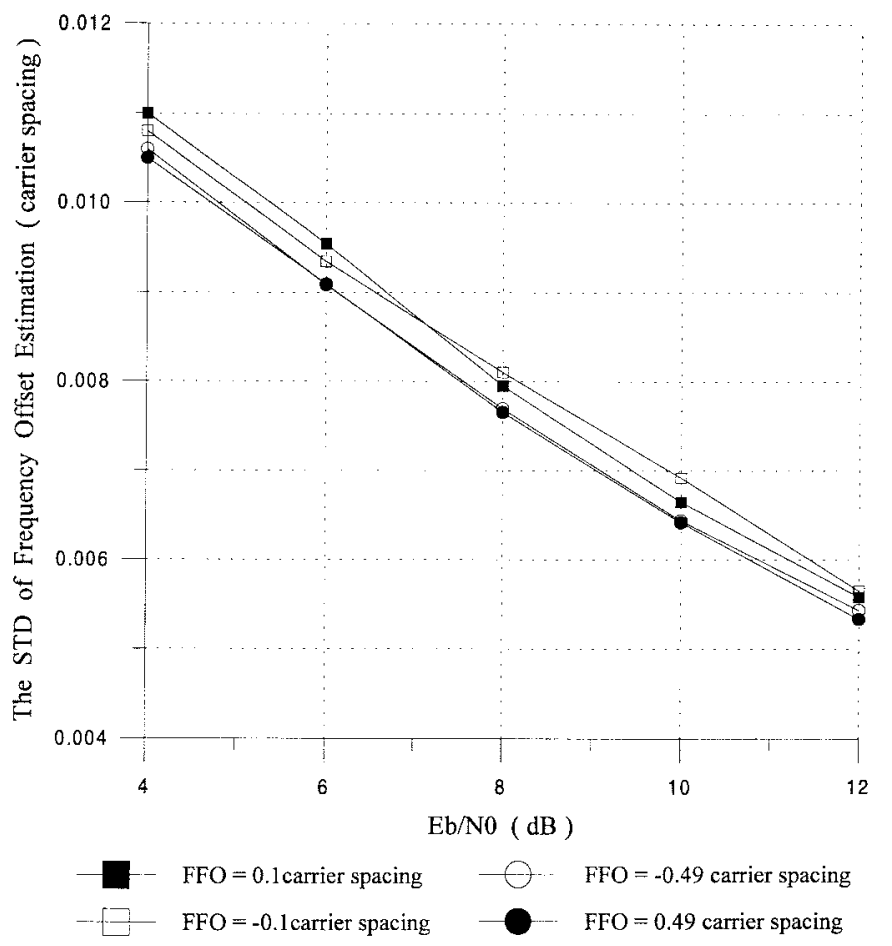

Fig. 7. The STD of frequency offset estimation in an AWGN channel.

The simulation results are shown in Fig. 10. The $(4,1,7)$ convolutional encoder used in this simulation is directly adopted from [4]. The code rate is $1 / 4$, and its constraint length is seven. The encoded bits are interleaved in frequency domain and then modulate each carrier of an Eureka 147 OFDM symbol using DQPSK. Again, we observe larger frequency offsets cause more BER degradation due to ICI. When frequency offset approaches 0.5 subcarrier spacing, the average BER approaches 0.5 .

The same two sets of FFO, as described in Section IV-A2, are used in simulation. The mean and STD of the fractional frequency offset estimation in a two-path fading channel is listed in Table IV and ploted in Fig. 11, respectively. We assume a $20 \mu$ s delay in the two-path fading channel in the simulation. Thus, $\Omega$ is about $10 \mu$ s, i.e. 20 samples. The last ten samples

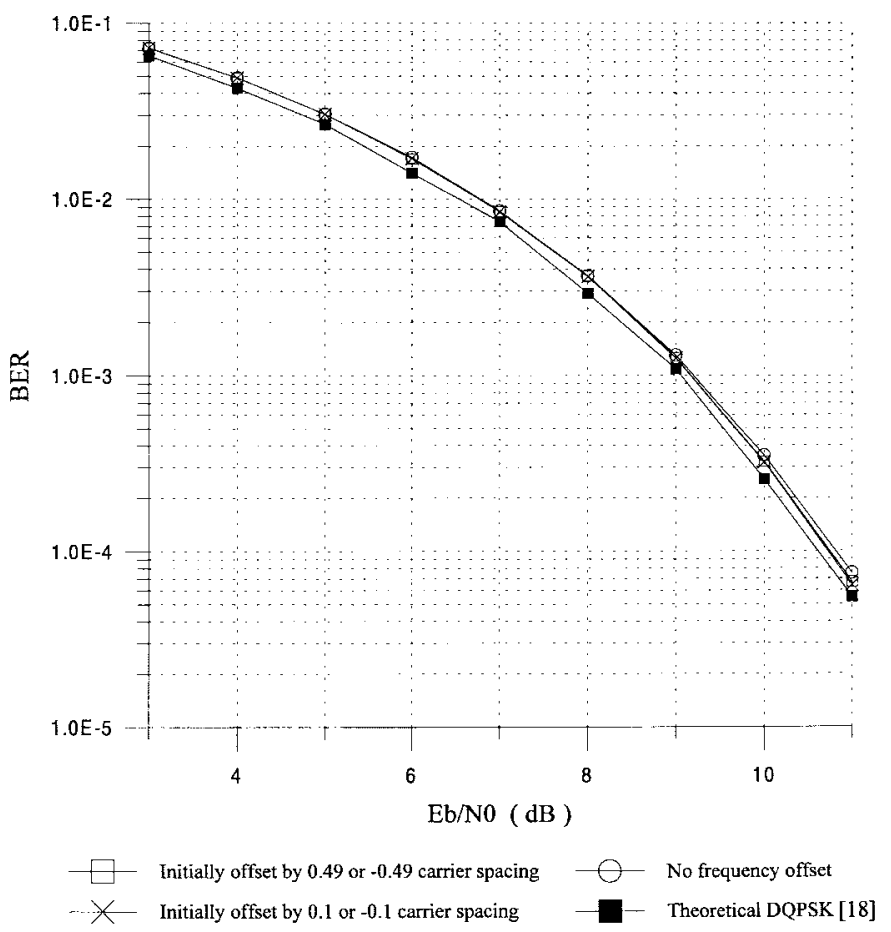

Fig. 8. BER performance with frequency offset compensation in an AWGN channel.

of $\Omega$ are processed in this simulation. From Table IV, we observe that the mean of fractional frequency offset estimation is very accurate in a two-path fading channel. It can also be observed from Fig. 11 that our algorithm achieves a much better accuracy than the needed 0.05 subcarrier spacing in a two-path fading channel, even when average SNR is down to $7 \mathrm{~dB}$.

The average BER performance of Eureka 147 mode 3 with our synchronization algorithm in a two-path fading channel is shown in Fig. 12. The $(4,1,7)$ convolutional encoder and the interleaver are the same as those used in Fig. 10. It can be seen that the BER performance with our synchronization algorithm almost coincides with the perfect synchronization case. The effectiveness of our synchronization algorithm can also be observed by comparing Fig. 12 with Fig. 10. 
TABLE III

Symbol Timing Estimation Results in a Two-Path Fading Channel. $\boldsymbol{\Omega}=(\mathrm{a}, \mathrm{b}), \mathrm{f}_{\mathrm{d}}=240 \mathrm{~Hz}$, Average SNR=13dB

\begin{tabular}{|c|c|c|c|c|c|c|c|}
\hline \multicolumn{2}{|c|}{ Multipath Delay } & $\begin{array}{c}5 \\
(105,158)\end{array}$ & $\begin{array}{c}10 \\
(115,158)\end{array}$ & $\begin{array}{c}15 \\
(125,158)\end{array}$ & $\begin{array}{c}20 \\
(135,158)\end{array}$ & $\begin{array}{c}25 \\
(145,158)\end{array}$ & $\begin{array}{c}30 \\
(155,158)\end{array}$ \\
\hline \multirow{3}{*}{$\begin{array}{l}\text { SWS }=63 \\
\text { (samples) }\end{array}$} & $\underset{\text { (i-th sample) }}{\widetilde{\mathrm{m}}}$ & 131.5 & 136.5 & 140.5 & 144.5 & 147.5 & 148.5 \\
\hline & $\begin{array}{c}\text { STD } \\
\text { (samples) }\end{array}$ & 3 & 4 & 5 & 6 & 7 & 9 \\
\hline & $\Delta_{l}, \Delta_{r}$ & $8.83,8.83$ & $5.38,5.38$ & $3.1,3.5$ & $1.58,2.25$ & $0.36,1.5$ & 0,0 \\
\hline \multirow{3}{*}{$\begin{array}{l}\text { SWS }=45 \\
\text { (samples) }\end{array}$} & $\underset{\text { (i-th sample) }}{\widetilde{m}}$ & 132.5 & 137.5 & 141.5 & 145.5 & 148.5 & 151.5 \\
\hline & $\begin{array}{c}\text { STD } \\
\text { (samples) }\end{array}$ & 2 & 3 & 4 & 5 & 6 & 9 \\
\hline & $\Delta_{l}, \Delta_{r}$ & $13.75,12.75$ & $7.5,6.83$ & $4.13,4.13$ & $2.1,2.5$ & $0.58,1.58$ & 0,0 \\
\hline \multirow{3}{*}{$\begin{array}{l}\text { SWS }=25 \\
\text { (samples) }\end{array}$} & $\widetilde{m}_{\text {(i-th sample) }}^{\widetilde{m}_{1}}$ & 132.5 & 137.5 & 141.5 & 146.5 & 150.5 & 153.5 \\
\hline & \begin{tabular}{|c|} 
STD \\
(samples) \\
\end{tabular} & 2 & 3 & 4 & 4 & 6 & 9 \\
\hline & $\Delta_{l}, \Delta_{r}$ & $13.75,12.75$ & $7.5,6.83$ & $4.13,4.13$ & $2.88,2.88$ & $0.92,1.25$ & 0,0 \\
\hline \multirow{3}{*}{$\begin{array}{c}\text { SWS }=5 \\
\text { (samples) }\end{array}$} & $\tilde{\mathrm{m}}_{\text {(i-th sample) }}$ & 133.5 & 137.5 & 142.5 & 147.5 & 151.5 & 156.5 \\
\hline & $\begin{array}{c}\mathrm{STD} \\
\text { (samples) }\end{array}$ & 3 & 3 & 4 & 5 & 6 & 8 \\
\hline & $\Delta_{l}, \Delta_{r}$ & $9.5,8.17$ & $7.5,6.83$ & $4.38,3.88$ & $2.5,2.1$ & $1.08,1.08$ & $0.19,0.19$ \\
\hline
\end{tabular}

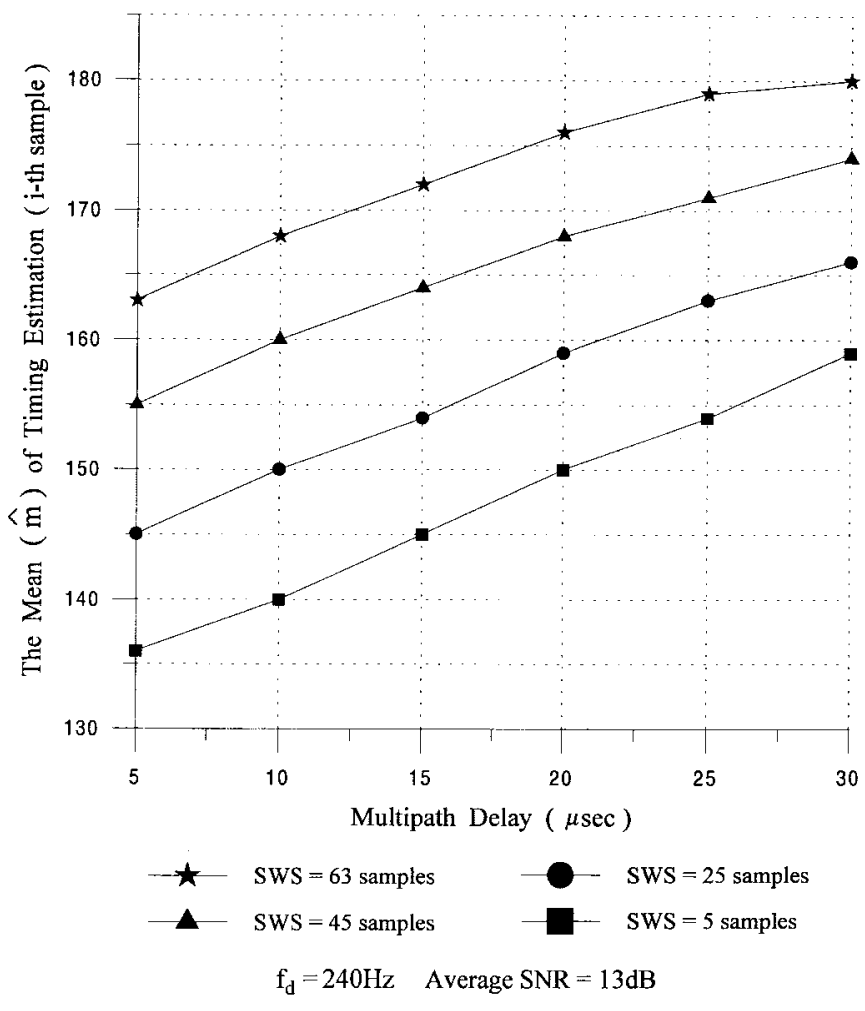

Fig. 9. The mean of an initial symbol timing estimation in a two-path fading channel. (Note: $\Gamma$ from ninety sixth to one hundred and fifty-eigth sample is assumed in this simulation.)

\section{Operations in a Single Frequency Network}

In contrast to FM broadcasting, Eureka 147 permits the operation of a single frequency network (SFN). In this network,

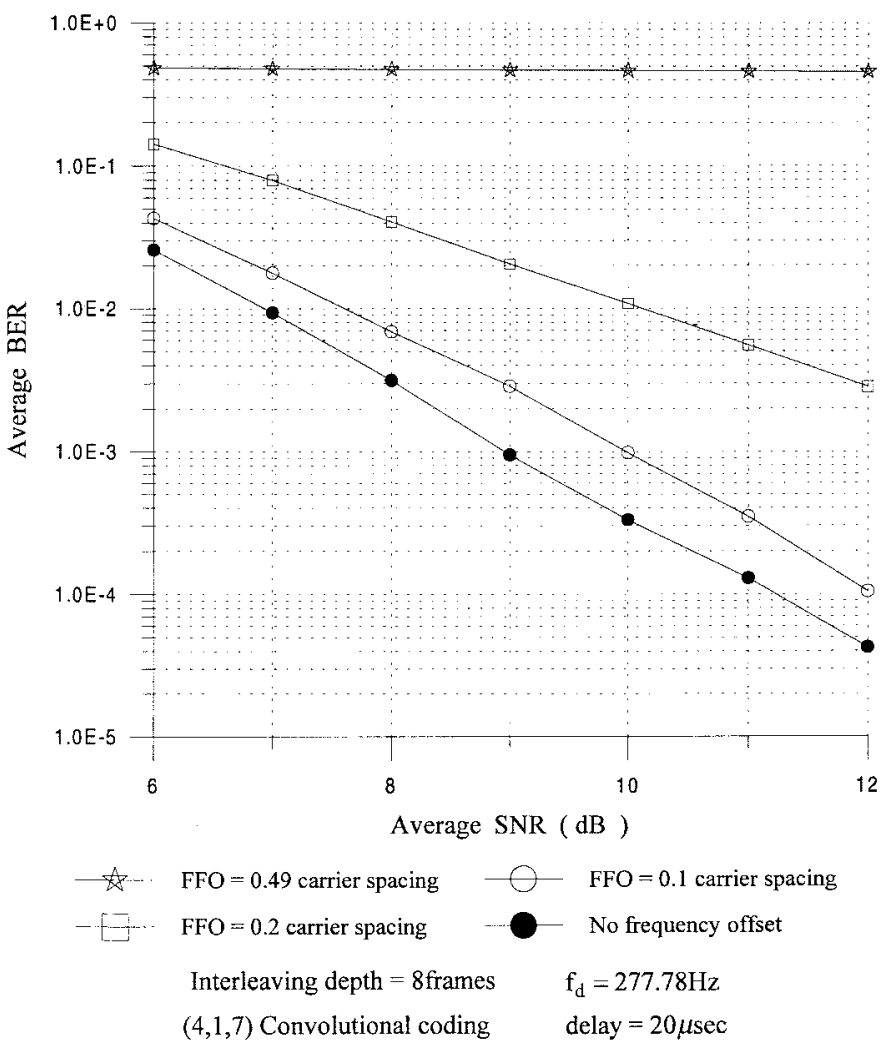

Fig. 10. Average BER performance with frequency offset as a parameter in a two-path fading channel.

there could be long delay and high level multipath signals coming from different transmitters. In this situation, an OFDM system needs to adopt a longer symbol duration and therefore 
TABLE IV

The Mean of Frequency Offset Estimation in a Two-Path Fading Channel. $f_{d}=240 H z$, Delay $=13 \mu$ Sec

\begin{tabular}{c|c|c|c|c|c}
\hline$\delta_{f r}^{\text {Average SNR }}(\mathrm{dB})$ & 7 & 9 & 11 & 13 & 15 \\
\hline 0.49 & 0.491 & 0.491 & 0.49 & 0.49 & 0.49 \\
\hline 0.1 & 0.0831 & 0.0881 & 0.0916 & 0.0947 & 0.0964 \\
\hline-0.1 & -0.0843 & -0.0895 & -0.0932 & -0.0955 & -0.097 \\
\hline-0.49 & -0.491 & -0.491 & -0.491 & -0.491 & -0.491 \\
\hline
\end{tabular}

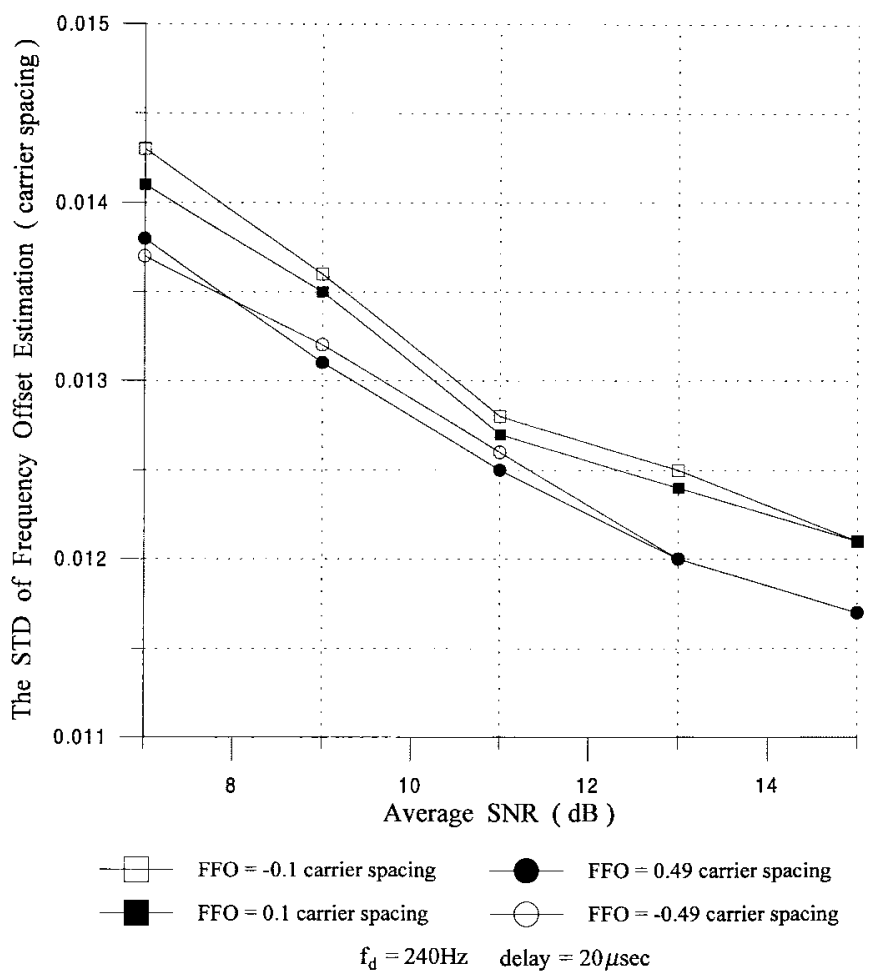

Fig. 11. The STD of frequency offset estimation in a two-path fading channel.

to provide a longer guard interval duration that can remove the effect of the distortion from long delay and high level echoes.

The simulation results demonstrated in the previous sections show that for mode 3 of the Eureka 147 system, our synchronization method obtains both symbol timing and frequency offset estimations accurately, as long as the multipath delay spread is less than $20 \mu \mathrm{s}$. The BER performance of our method also approaches to the ideal synchronization case, as can be observed in Fig. 12. The length of a guard interval duration is hence the most important parameter for an OFDM system to operate successfully in a multipath fading environment. With a larger multipath delay spread, either mode 2 or mode 1 of Eureka 147 can be used to achieve an SFN. For example, in mode 1 of Eureka 147, its symbol duration is $1.246 \mathrm{~ms}$, and the

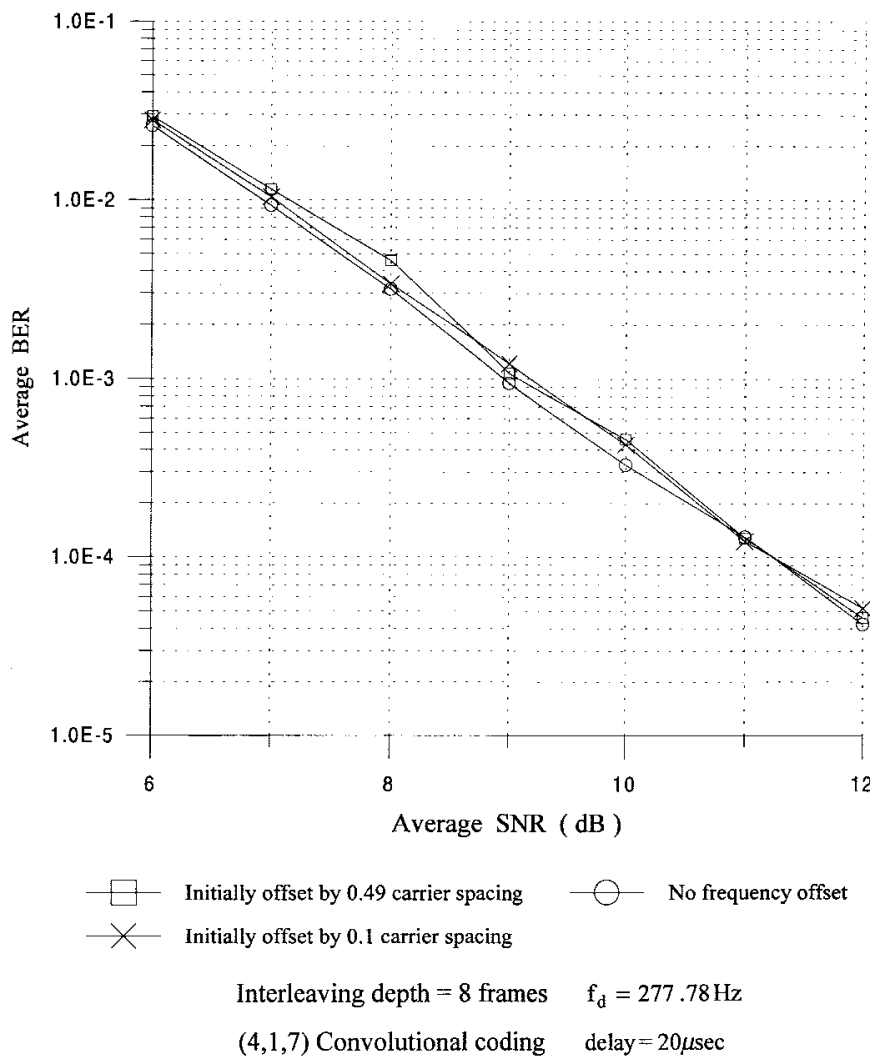

Fig. 12. Average BER performance after frequency offset compensation in a two-path fading channel.

guard interval duration is $246 \mu \mathrm{s}$; both parameters are about eight times that of the corresponding parameters of mode 3 . With mode 1, our method will be still workable because the signal format of mode 1 is the same as mode 3 . Therefore, our synchronization method can be used effectively in an SFN.

\section{CONClusion}

The main technologies of DAB, particularly the OFDM transmission scheme, are very useful in broadband wireless transmission. This paper presents a method that jointly achieves symbol, carrier, and frame synchronization for an 
OFDM signal. In this method, we estimate symbol timing first and then locate a corresponding multipath interference free period to calculate the fractional frequency offset. After compensating for this fractional frequency offset, we estimate integral frequency offset. This method is shown to perform well in an AWGN channel when $E_{b} / N_{0}$ is down to $4 \mathrm{~dB}$. The method is also robust in a two-path fading channel. Our simulation results suggest that the system BER degradation with our synchronization method is negligible as compared with the ideal synchronization case. This method can also be used in other applications, such as DVB and HIPERLAN/2.

\section{REFERENCES}

[1] R. W. Chang, "Synthesis of band-limited orthogonal signals for multichannel data transmission," Bell Syst. Tech. J., vol. 45, pp. 1775-1796, Dec. 1966.

[2] R. W. Chang and R. A. Gibbey, "A theoretical study of performance of an orthogonal multiplexing data transmission scheme," IEEE Trans. Commun. Technol., vol. COM-16, pp. 529-540, Aug. 1968.

[3] S. B. Weinstein and P. M. Ebert, "Data transmission by frequencydivision multiplexing using the discrete Fourier transform," IEEE Trans. Commun., vol. COM-19, pp. 628-634, Oct. 1971.

[4] European Telecommunication Standard, "Digital audio broadcasting (DAB) to mobile, portable, and fixed receivers," ETS 300 401, Feb. 1995.

[5] B. Le Floch, R. Halbert-Lassalle, and D. Castelain, "Digital sound broadcasting to mobile receivers," IEEE Trans. Consum. Electron., vol. 35, pp. 457-462, Aug. 1989.

[6] Y. Wu and W. Y. Zou, "Performance simulation of COFDM for TV broadcast application," SMPTE J., pp. 258-265, May 1995.

[7] H. Sari, G. Karam, and I. Jeanclaude, "Transmission techniques for digital terrestrial TV broadcasting," IEEE Commun. Mag., vol. 2, pp. 100-109, Feb. 1995.

[8] A. Dekorsy and K.-D. Kammeyer, "M-ary orthogonal modulation for multi-carrier spread-spectrum uplink transmission," in Proc. IEEE Int. Conf. Communications (ICC'98), vol. 2, pp. 1004-1008.

[9] J.-J. van de Beek, M. Sandell, M. Isaksson, and P. O. Borjesson, "Lowcomplex frame synchronization in OFDM systems," in Proc. IEEE Int Conf. Universal Personal Communications, Oct. 1995, pp. 982-986.

[10] F. Classen and H. Meyr, "Frequency synchronization algorithms for OFDM systems suitable for communication over frequency selective fading channels," in Proc. ICC'94, pp. 13.1655-13.1659.

[11] H. Daffara and A. Chouly, "Maximum likelihood frequency detectors for orthogonal multicarrier systems," in Proc. ICC'93, pp. 766-771.

[12] H. Daffara and O. Adami, "A new frequency detector for orthogonal multicarrier transmission techniques," in 45th IEEE Vehicular Technology Conf., 1995, pp. 804-809.

[13] P. H. Moose, "A technique for orthogonal frequency division multiplexing frequency offset correction," IEEE Trans. Commun., vol. 42, pp. 2908-2914, Oct. 1994.

[14] W. D. Warner and C. Leung, "OFDM/FM frame synchronization for mobile radio data communication," IEEE Trans. Vehic. Technol., vol. 42, pp. 302-313, Aug. 1993.

[15] Y.-L. Huang, C.-C. Lu, and C.-C. Huang, "Synchronization system of digital audio broadcasting (DAB) receiver," in Proc. IEEE Int. Conf. Consumer Electronics, June 1997, pp. 370-371.

[16] K. Taura et al., "A digital audio broadcasting (DAB) receiver," IEEE Trans. Consumer Electron, vol. 42, pp. 322-326, Aug. 1996

[17] C.-R. Sheu, Y.-L. Huang, and C.-C. Huang, "Joint symbol, frame, and carrier synchronization for Eureka 147 DAB system," in Proc. IEEE Int. Conf. Universal Personal Communications, Oct. 1997, pp. 1313.693-1313.697.

[18] A. V. Oppenheim and R. W. Schafer, Digital Signal Processing. Englewood Cliffs, NJ: Prentice-Hall, 1975.
[19] P. Shelswell, "The COFDM modulation system: The heart of digital audio broadcasting," Electron. Commun. Eng. J., pp. 127-136, June 1995.

[20] M. Basseville and I. V. Nikiforov, Eds., Detection of Abrupt Changes: Theory and Applications. Englewood Cliffs, NJ: PTR Prentice-Hall, 1993.

[21] J. G. Proakis, Digital Communications, 3rd ed. New York: McGrawHill, 1995.

[22] T. Pellet, M. Van Bladel, and M. Moeneclaey, "EER sensitivity of OFDM Systems to carrier frequency offset and wiener phase noise," IEEE Trans. Commun., vol. 43, pp. 191-193, Feb./Mar./Apr. 1995.

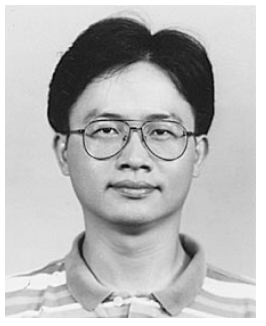

Yung-Liang Huang (S'93-M'99) was born in Taipei, Taiwan, R.O.C. He received the B.S. and M.S. degrees in communications from the National Chiao Tung University (NCTU), Hsinchu, Taiwan, R.O.C., in 1985 and 1987, respectively. He is currently pursuing the Ph.D. degree at NCTU.

In 1987, he joined Electronics Research \& Services Organizations (ERSO) and later the Computer \& Communication Laboratories (CCL), both at the Industrial Technology Research Institute (ITRI), where he was involved in the developments of V.32 modem data pump, video compression, DECT, and Eureka 147 DAB systems. He was the Project Leader of both the video compression and DECT projects. Since 1998, he has been with the Solomon Wireless Tech. Corp., Taipei, Japan, as a Senior Project Manager, working on various frequency-hopping communication systems. His interests include the design and implementation of communication systems and spread spectrum systems for wireless communications.

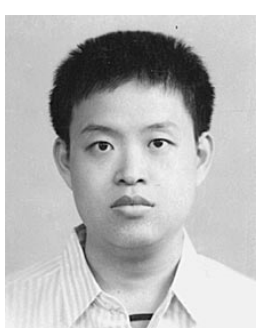

Chorng-Ren Sheu (S'96) was born in Kaohsiung, Taiwan, R.O.C. He received the B.S. and M.S degrees in communication engineering from the National Chiao Tung University (NCTU), Hsinchu, Taiwan, R.O.C., in 1994 and 1996, respectively. He is currently pursuing the $\mathrm{Ph} . \mathrm{D}$ degree in communication engineering at NCTU.

His research interests include OFDM and personal communications.

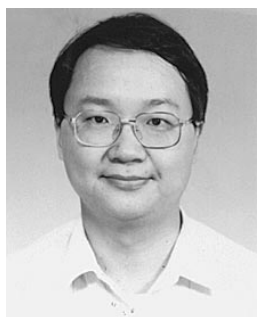

Chia-Chi Huang was born in Taiwan, R.O.C. He received the B.S. degree in electrical engineering from National Taiwan University in 1977, and the M.S. and Ph.D. degrees in electrical engineering from the University of California, Berkeley, in 1980 and 1984, respectively.

From 1984 to 1988, he was an RF and Communication System Engineer with the Corporate Research and Development Center, General Electric Co., Schenectady, NY, where he worked on mobile radio communications. From 1989 to 1992, he was with the IBM T. J. Watson Research Center, Yorktown Heights, NY, as a Research Staff Member, working on indoor radio communications. Since September 1992, he has been with the Department of Communication Engineering, National Chiao Tung University, Hsinchu, Taiwan, R.O.C., as an Associate Professor. 